\title{
樟脑磺酸噻唑腙类化合物的设计、合成及抗氧化应用
}

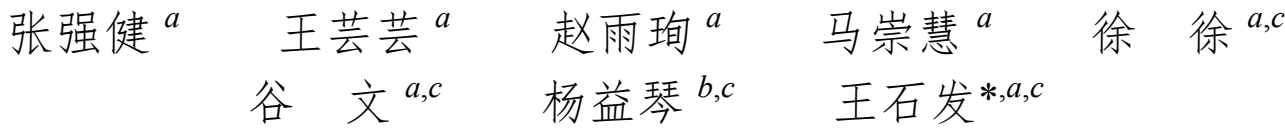 \\ ( ${ }^{a}$ 南京林业大学化学工程学院 南京 210037) \\ ( $b$ 南京林业大学轻工与食品学院 南京 210037) \\ ( ${ }^{c}$ 南京林业大学林业资源高效加工利用协同创新中心 南京 210037)
}

\begin{abstract}
摘要 以樟脑衍生物樟脑磺酸为原料, 经缩合、环化等反应, 合成了 22 个樟脑磺酸噻唑腙类化合物, 采用 ${ }^{1} \mathrm{H} \mathrm{NMR} 、{ }^{13} \mathrm{C}$ NMR、HR-MS 等方法对产物的结构进行表征, 并研究了它们的抗氧化活性. 结果表明, 不同取代基的樟脑磺酸噻唑腙 类化合物表现出不同的抗氧化活性，其中(2-(2-(4-(4-氰基苯基)噻唑-2-基)亚肼基)-7,7-二甲基双环[2.2.1]庚-1-基)甲磺酸 (Q19)消除 1,1-二苯基-2-三硝基苯胇(DPPH)自由基的 $\mathrm{IC}_{50}$ 值可达到 $176 \mu \mathrm{mol} / \mathrm{L},(2-(2-(4-(4$-氟苯基)噻唑-2-基)亚肼 基)-7,7-二甲基双环 [2.2.1]庚-1-基)甲磺酸(Q3)消除 2,2-联氮-二(3-乙基-苯并噻唑-6-磺酸)二铵盐(ABTS)自由基的 $\mathrm{IC}_{50}$ 值 可达到 $20.6 \mu \mathrm{mol} / \mathrm{L},(E)$-(7,7-二甲基-2-(2-(4-(3-甲苯基)噻唑-2-基)亚胖基)双环[2.2.1]庚-1-基)甲磺酸( $\mathbf{Q 8}$ )对羟基自由基 的消除率可达到 66.2\%, (2-(2-(4-(4-联苯基)噻唑-2-基)亚肼基)-7,7-二甲基双环 2.2 .1$]$ 庚-1-基)甲磺酸(Q20)消除过氧自由 基的 $\mathrm{IC}_{50}$ 值可达到 $20.7 \mu \mathrm{mol} / \mathrm{L}$, 均远超过阳性对照 trolox 的抗氧化活性. (2-(2-(4-(2-羟基苯基)噻唑-2-基)亚胇基)-7,7二甲基双环[2.2.1]庚-1-基)甲磺酸(Q16)抑制酪氨酸酶的 $\mathrm{IC}_{50}$ 值可达 $154.9 \mu \mathrm{mol} / \mathrm{L}$ ，也优于阳性对照曲酸. 对樟脑磺酸基 噻唑腙类化合物的抗氧化机理进行了探索.
\end{abstract}

关键词＼cjkstart樟脑磺酸; 噻唑腙; 抗氧化活性

\section{Design, Synthesis and Antioxidant Application of Camphorsulfonic Acid Thiazolylhydrazone Derivatives}

\author{
Zhang, Qiangjian ${ }^{a}$ \\ Wang, Yunyun ${ }^{a}$ \\ Zhao, Yuxun ${ }^{a}$ \\ Ma, Chonghui ${ }^{a}$ \\ $\mathrm{Xu}, \mathrm{Xu}^{a, c}$ \\ Gu, Wen ${ }^{a, c} \quad$ Yang, Yiqing ${ }^{b, c} \quad$ Wang, Shifa ${ }^{*, a, c}$ \\ ( ${ }^{a}$ College of Chemical Engineering, Nanjing Forestry University, Nanjing 210037) \\ ( ${ }^{b}$ College of Light Industry and Food, Nanjing Forestry University, Nanjing 210037) \\ ( ${ }^{c}$ Co-Innovation Center of Efficient Processing and Utilization of Forest Resources, \\ Nanjing Forestry University, Nanjing 210037)
}

\begin{abstract}
A series of camphorsulfonic acid thiazolylhydrazone derivatives were synthesized by using camphorsulfonic acid derivated from natural camphor as the starting material in three steps, including condensation with aminothiourea and cyclization with bromoacetophenone. Their structures were characterized by ${ }^{1} \mathrm{H}$ NMR, ${ }^{13} \mathrm{C}$ NMR and HR-MS, and their antioxidant activities were also investigated. The results showed that these compounds had good antioxidant activities compared with the positive control trolox. Among of them, (2-(2-(4-(4-cyanophenyl)thiazol-2-yl)indenyl)-7,7-dimethylbicyclo[2.2.1]hept-1-yl)methanesulfonic acid (Q19) exhibited the relatively strong 1,1-diphenyl-2-picrylhydrazyl (DPPH) radical scavenging activity with $\mathrm{IC}_{50}$ value of $176 \mu \mathrm{mol} / \mathrm{L}$, (2-(2-(4-(4-fluorophenyl)thiazol-2-yl)hydrazino)-7,7-dimethylbicyclo[2.2.1] hept-1-yl)methanesulfonic acid (Q3) exhibited the relatively strong diammonium 2,2'-azino-bis(3-ethylbenzothiazoline6-sulfonate) (ABTS) radical scavenging activity with $\mathrm{IC}_{50}$ value of $20.6 \mu \mathrm{mol} / \mathrm{L},(E)-(7,7-$ dimethyl-2-(2-(4-(3-methyl)-thiazol2-yl)indenyl)bicyclo[2.2.1]hept-1-yl)-sulfonic acid (Q8) exhibited the strong hydroxyl radical scavenging activity with scavenge rate 66.2\%, (2-(2-(4-(4-biphenyl)thiazol-2-yl)indenyl)-7,7-dimethylbicyclo[2.2.1]hept-1-yl)methane-sulfonic acid (Q20) exhibited the relatively strong superoxide radical scavenging activity with $\mathrm{IC}_{50}$ value of $20.7 \mu \mathrm{mol} / \mathrm{L}$. Compared with the posi-
\end{abstract}

* Corresponding author. E-mail: wangshifa65@163.com

Received January 30, 2019; revised March 17, 2019; published online April 11, 2019.

Project supported by the National Natural Science Foundation of China (No. 31470592).

国家自然科学基金(No. 31470592)资助项目. 
tive control kojic acid, (2-(2-(4-(2-hydroxyphenyl)thiazol-2-yl)arylene)-7,7-dimethylbicyclo[2.2.1]hept-1-yl)methanesulfonic acid (Q16) exhibited remarkable tyrosinase inhibitory activity with $\mathrm{IC}_{50}$ value of $154.9 \mu \mathrm{mol} / \mathrm{L}$. It was known from the structure-activity relationship that the structure of $\mathrm{R}$ gave great influence on the activities of thiazolylhydrazone derivatives.

Keywords camphorsulfonic acid; thiazolylhydrazone; antioxidant activity

据有关研究报道 ${ }^{[1,2]}$, 人体衰老是一种复杂的生化 进程, 而皮肤老化在识别人体衰老方面起着重要的作 用. 皮肤老化有两种类型: 一种是时间老化, 皮肤结构 和功能随着时间逐渐恶化; 另一种是外源性老化, 即光 老化, 皮肤纹理因长期暴露在阳光下而产生变化 ${ }^{[3,4]}$. 而 自由基和活性氧是促进皮肤老化的最重要成分, 它们在 皮肤细胞中会诱导氧化应激 ${ }^{[5]}$, 氧化应激易对脂质、蛋 白质和 DNA 等大分子造成损害, 进而导致许多慢性疾 病, 例如炎症、动脉硬化、糖尿病、癌症、心血管和神 经系统退行性疾病, 如阿尔茨海默症(Alzheimer's disease, AD)与血管性痴呆(Vascular dementia, Va D)等 ${ }^{[6,7]}$. 此外, 食物中脂质的氧化会导致令人不愉快的腐臭味或 异 味 ${ }^{[8]}$, 因此, 开发研制具有高抗氧化活性的化合物 成为科学家们的主流研究方向.

近年来, 关于抗氧化剂的研究越来越多, 对天然产 物改性来制备抗氧化剂更是研究热点. Amorati 等 ${ }^{[9]}$ 提取 了一种多低沸点组分的精油, 利用其中酚类成分的活性 来防止脂质氧化. 但是由于其易挥发的特性, 精油的抗 氧化作用在高温下受到极大的限制. Liu 等 ${ }^{[10]}$ 采用热水 提取法得到一种冬瓜多糖, 它是通过 $\beta$-糖苗键连接的多 种单糖(包括葡萄糖、半乳糖、甘露糖、木糖、阿拉伯 糖、鼠李糖), 实验表明, 冬瓜多糖对超氧阴离子自由基 具有较好的清除能力. Didem 等 ${ }^{[11]}$ 篮选了一种类黄酮化 合物, 表现出了良好的酪氨酸酶抑制性, 对氧化应激反 应有较好的抑制作用. 此外, 在天然化合物的研究中, 常用直接消除自由基或提供还原能力以抵消自由基引 起的氧化应激来表征抗氧化剂的生物活性效果. 因此, 常用测定 2,2'-联氮双(3-乙基苯并噻唑啉-6-磺酸)二铵盐 (ABTS)自由基消除能力 ${ }^{[12]}, 1.1$-二苯基-2-苦肼基(DPPH) 自由基消除能力 ${ }^{[13]}$, 过氧自由基消除(Peroxyl Radical Scavenging Capacity, PSC) 实验 $^{[14]}$ 以及 Fenton 反应 ${ }^{[15]}$ 中 抑制剂提供的还原能力来表征噻唑腙类化合物的抗氧 化活性, 使用酪氨酸酶的抑制效果 ${ }^{[16]}$ 来评估化合物对 氧化应激效果的减弱, 期望以此为抗氧化药物及化妆品 的发展提供帮助.

樟脑是一种双环单萜酮类物质，具有抑菌、驱虫、 消炎、镇痛等多种生物活性, 几个世纪以来一直应用于 精油制剂以及传统医学, 如今更多地将其进行改性, 应 用到食品加工、香料和药物方面 ${ }^{[17-21]}$, 而且樟脑衍生物 还表现出杀菌、除草以及抗肿瘤等多方面的生物活性. 噻唑环是一种含有氮硫的五元不饱和杂环, 是常见的具
有药学活性的物质. 例如磺胺噻唑、博来霉素、噻唑弗 林等均为噻唑环框架的药物. 噻唑类化合物在过敏症 ${ }^{[22]}$ 、高血压 ${ }^{[23]} 、$ 炎症 ${ }^{[24]}$ 、精神分裂症 ${ }^{[25]} 、$ 细菌感染 ${ }^{[26]} 、$ 失眠症 ${ }^{[27]}$. HIV 感染 ${ }^{[28]}$ 以及血栓 ${ }^{[29]}$ 等疾病治疗方面发挥 了重要的作用. 在设计抗氧化剂方面, 以噻唑环作为活 性框架已受到许多研究者的关注 ${ }^{[30 ~ 32] . ~}$. 为寻找具有高 效抗氧化活性的化合物, 本文以天然樟脑衍生物樟脑磺 酸为先导化合物, 并结合课题组前期的工作基础 ${ }^{[33 \sim 37]}$, 经缩合、环化等反应, 设计合成了 22 个新型的樟脑磺酸 基噻唑腙类化合物, 采用 ${ }^{1} \mathrm{H} N M R 、{ }^{13} \mathrm{C} N M R 、 H R M S$ 等方法对其结构进行表征, 研究了噻唑腙类化合物的抗 氧化活性. 结果表明, 化合物在多方面均表现出优良的 的抗氧化活性，其中化合物 Q19 消除 DPPH 自由基的 $\mathrm{IC}_{50}$ 值可达到 $176 \mu \mathrm{mol} / \mathrm{L}$, 化合物 $\mathbf{Q 3}$ 消除 ABTS 自由 基的 $\mathrm{IC}_{50}$ 值可达 $20.6 \mu \mathrm{mol} / \mathrm{L}$ ，化合物 $\mathbf{Q 8}$ 对羟基自由基 的消除率可达到 66.2\%, 化合物 Q20 消除过氧自由基的 $\mathrm{IC}_{50}$ 值可达 $20.7 \mu \mathrm{mol} / \mathrm{L}$, 均远超过 trolox 的抗氧化活性. 化合物 Q16 抑制酪氨酸酶的 $\mathrm{IC}_{50}$ 值可达 $154.9 \mu \mathrm{mol} / \mathrm{L}$, 也优于阳性对照曲酸.

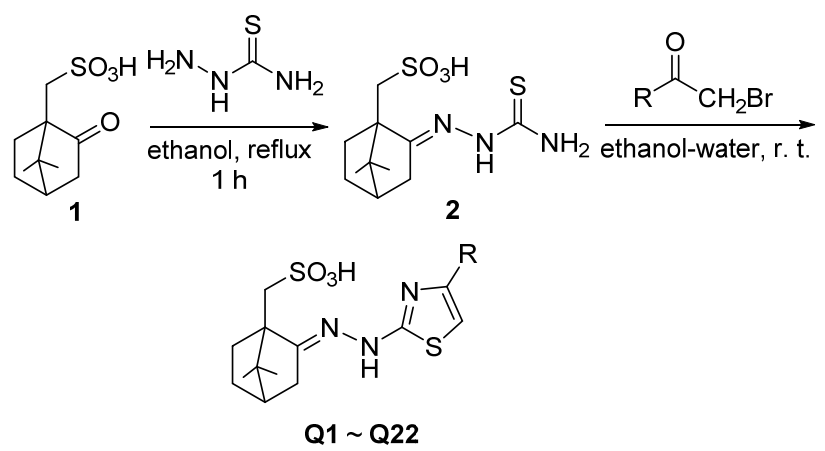

Q1: $\mathrm{R}=2-\mathrm{FC}_{6} \mathrm{H}_{4}, \mathbf{Q 2}: \mathrm{R}=3-\mathrm{FC}_{6} \mathrm{H}_{4}$, Q3: $\mathrm{R}=4-\mathrm{FC}_{6} \mathrm{H}_{4}, \mathbf{Q 4}: \mathrm{R}=$ 2- $\mathrm{CIC}_{6} \mathrm{H}_{4}, \mathbf{Q 5 :} \mathrm{R}=3-\mathrm{ClC}_{6} \mathrm{H}_{4}, \mathbf{Q 6 :}: \mathrm{R}=4-\mathrm{ClC}_{6} \mathrm{H}_{4}, \mathbf{Q 7}: \mathrm{R}=2-\mathrm{CH}_{3} \mathrm{C}_{6} \mathrm{H}_{4}$, Q8: $\mathrm{R}=3-\mathrm{CH}_{3} \mathrm{C}_{6} \mathrm{H}_{4}, \mathbf{Q 9 :} \mathrm{R}=4-\mathrm{CH}_{3} \mathrm{C}_{6} \mathrm{H}_{4}, \mathbf{Q 1 0}: \mathrm{R}=2-\mathrm{CH}_{3} \mathrm{OC}_{6} \mathrm{H}_{4}$, Q11: $\mathrm{R}=3-\mathrm{CH}_{3} \mathrm{OC}_{6} \mathrm{H}_{4}$, Q12: $\mathrm{R}=4-\mathrm{CH}_{3} \mathrm{OC}_{6} \mathrm{H}_{4}$, Q13: $\mathrm{R}=2-\mathrm{O}_{2} \mathrm{~N}-$ $\mathrm{C}_{6} \mathrm{H}_{4}$, Q14: $\mathrm{R}=3-\mathrm{O}_{2} \mathrm{NC}_{6} \mathrm{H}_{4}$, Q15: $\mathrm{R}=4-\mathrm{O}_{2} \mathrm{NC}_{6} \mathrm{H}_{4}$, Q16: $\mathrm{R}=2-\mathrm{HO}-$ $\mathrm{C}_{6} \mathrm{H}_{4}, \mathrm{Q} 17: \mathrm{R}=3-\mathrm{HOC}_{6} \mathrm{H}_{4}, \mathrm{Q18}: \mathrm{R}=4-\mathrm{HOC}_{6} \mathrm{H}_{4}, \mathrm{Q} 19: \mathrm{R}=4-\mathrm{CN}-$ $\mathrm{C}_{6} \mathrm{H}_{4}$, Q20: $\mathrm{R}=4-\mathrm{C}_{6} \mathrm{H}_{5} \mathrm{C}_{6} \mathrm{H}_{4}$, Q21: $\mathrm{R}=2-\mathrm{C}_{10} \mathrm{H}_{7}$, Q22: $\mathrm{R}=\mathrm{C}_{6} \mathrm{H}_{5}$

图式 1 樟脑磺酸塞唑腙类衍生物的合成 Scheme 1 Synthesis of camphorsulfonic acid thiazolylhydrazone derivatives $\mathbf{Q 1} \sim \mathbf{Q 2 2}$

\section{1 结果与讨论}

\section{1 合成与表征}

由樟脑磺酸合成噻唑类衍生物 $\mathbf{Q 1} \mathbf{Q 2 2}$ 的方案包 
括两个步骤: 加成缩合和 Hantzch 成环反应. 樟脑磺酸 (1) 与氨基硫脲缩合制得中间体 2, 中间体 2 与溴代苯乙 酮进行环化制得噻唑腙类化合物 $\mathbf{Q 1} \sim \mathbf{Q 2 2}$. 通过 ${ }^{1} \mathrm{H}$ $\mathrm{NMR},{ }^{13} \mathrm{C} \mathrm{NMR}, \mathrm{HRMS}$ 表征目标化合物的结构. 例如 由 $\mathbf{Q 1}$ 的 ${ }^{1} \mathrm{H}$ NMR 分析结果表明, 在 $\delta 7.23 \sim 7.96$ 处观 察到对应芳族质子的单峰和双峰, 在 $\delta 7.19$ 处发现了噻 唑环上的质子峰. Q1 的 ${ }^{13} \mathrm{C} \mathrm{NMR}$ 显示芳族碳的峰在 $\delta$ $108.86 \sim 161.02$ 处, 且 $\delta 116.53,129.22$ 和 161.02 处存在 碳氟共轭峰, $\delta$ 108.70, 164.3 和 170.60 处的峰表明了噻 唑环的存在.

\section{2 抗氧化活性}

化合物 Q1 $\sim \mathbf{Q 2 2}$ 的抗氧化活性测试数据见表 1. 由 表 1 数据可知, 化合物 2 在各个实验中均没表现出明显 的抗氧化活性, 表明衍生化后的噻唑腙结构对化合物 Q1 Q22 的抗氧化活性起着决定性的作用. Q14, Q1 和 Q19三个化合物在消除 DPPH 自由基方面表现出显著的 活性, 其中 Q19 的活性最强, 其消除 DPPH 自由基的 $\mathrm{IC}_{50}$ 值为 $176.0 \mu \mathrm{mol} / \mathrm{L}$, 效果强于阳性对照 trolox 的 $\mathrm{IC}_{50}$ 值 $320.2 \mu \mathrm{mol} / \mathrm{L}$. 在消除 ABTS 自由基方面, 噻唑腙类 化合物大多均表现出良好的活性, 效果优于阳性对照 trolox 的 $\mathrm{IC}_{50}$ 值 $65.49 \mu \mathrm{mol} / \mathrm{L}$, 其中 $\mathbf{Q 3}$ 的活性最强, 其
消除 $\mathrm{ABTS}$ 自由基的 $\mathrm{IC}_{50}$ 值为 $20.61 \mu \mathrm{mol} / \mathrm{L}$, 约为阳性 对照 trolox 的 3.18 倍. 在消除过氧自由基方面, 大多数 化合物都表现出了较好的活性，其中 Q20 表现出了最好 的活性, 它的 $\mathrm{IC}_{50}$ 值为 $20.7 \mu \mathrm{mol} / \mathrm{L}$, 活性约为阳性对照 trolox 的 6.76 倍. 化合物 Q16 抑制酪氨酸酶的 $\mathrm{IC}_{50}$ 值可 达 $154.9 \mu \mathrm{mol} / \mathrm{L}$, 也优于阳性对照曲酸. 噻唑腙类化合 物对着基自由基的清除率结果见图 1, Q1, Q2, Q4, Q8 和 Q13 均表现出优于阳性对照 trolox 的抗氧化活性. 尤 其是 Q8，对羟基自由基的消除率高达 66.2\%.

\section{3 化合物清除 DPPH 自由基的反应机理}

噻唑腙对 DPPH 自由基的清除活性与腙部分中的 $\mathrm{NH}$ 活性基团的存在有关，该部分具有提供氢原子以消 除 DPPH 自由基的能力 ${ }^{[38]}$. 由图 2 所示, $m / z 449.1021$ 为 化合物 Q15 的脱质子离子峰, $m / z 394.0849$ 为 DPPH 自 由基得到一个质子后的脱质子离子峰, $m / z 226.0011$ 可 能是 DPPH 自由基中三硝基苯胺的分子碎片峰，且质谱 图中未见明显的化合物 $\mathbf{Q 1 5}$ 与 $\mathrm{DPPH}$ 相互加和的分子 峰. 因此推测樟脑磺酸噻唑腙类化合物对 DPPH 自由基 的消除机理可能是质子转移，其反应机理如 Scheme 2 所示.

表 1 化合物 Q1 $\sim \mathbf{Q 2 2}$ 的抗氧化活性

Table1 Antioxidant activity of compounds $\mathbf{Q 1} \sim \mathbf{Q 2 2}$

\begin{tabular}{|c|c|c|c|c|c|}
\hline \multirow{2}{*}{ Compound } & \multirow{2}{*}{$\mathrm{R}$} & \multicolumn{4}{|c|}{$\mathrm{IC}_{50} /\left(\mu \mathrm{mol} \cdot \mathrm{L}^{-1}\right)$} \\
\hline & & DPPH & ABTS & PSC & Tyrosinase \\
\hline 2 & - & $>1000$ & $>100$ & $>200$ & $>1000$ \\
\hline Q1 & $2-\mathrm{FC}_{6} \mathrm{H}_{4}$ & $>1000$ & $29.4 \pm 0.1$ & $105.8 \pm 0.2$ & $>1000$ \\
\hline Q2 & $3-\mathrm{FC}_{6} \mathrm{H}_{4}$ & $>1000$ & $26.4 \pm 0.1$ & $66.5 \pm 0.1$ & $264.5 \pm 0.3$ \\
\hline Q3 & 4- $\mathrm{FC}_{6} \mathrm{H}_{4}$ & $>1000$ & $20.6 \pm 0.1$ & $47.1 \pm 0.1$ & $308.0 \pm 0.4$ \\
\hline Q4 & $2-\mathrm{ClC}_{6} \mathrm{H}_{4}$ & $506.0 \pm 0.1$ & $34.7 \pm 0.1$ & $145.7 \pm 0.2$ & $>1000$ \\
\hline Q5 & $3-\mathrm{ClC}_{6} \mathrm{H}_{4}$ & $526.3 \pm 0.2$ & $28.6 \pm 0.1$ & $64.2 \pm 0.1$ & $266.4 \pm 0.2$ \\
\hline Q6 & 4- $\mathrm{ClC}_{6} \mathrm{H}_{4}$ & $845.7 \pm 0.5$ & $29.9 \pm 0.1$ & $53.4 \pm 0.1$ & $317.4 \pm 0.3$ \\
\hline Q7 & 2- $\mathrm{CH}_{3} \mathrm{C}_{6} \mathrm{H}_{4}$ & $702.6 \pm 0.4$ & $33.6 \pm 0.1$ & $59.7 \pm 0.1$ & $>1000$ \\
\hline Q8 & $3-\mathrm{CH}_{3} \mathrm{C}_{6} \mathrm{H}_{4}$ & $>1000$ & $>100$ & $>200$ & $675.19 \pm 0.5$ \\
\hline Q9 & 4- $\mathrm{CH}_{3} \mathrm{C}_{6} \mathrm{H}_{4}$ & $353.9 \pm 0.2$ & $33.2 \pm 0.1$ & $66.2 \pm 0.1$ & $>1000$ \\
\hline Q10 & $2-\mathrm{CH}_{3} \mathrm{OC}_{6} \mathrm{H}_{4}$ & $476.5 \pm 0.2$ & $29.4 \pm 0.1$ & $30.6 \pm 0.1$ & $>1000$ \\
\hline Q11 & $3-\mathrm{CH}_{3} \mathrm{OC}_{6} \mathrm{H}_{4}$ & $749.7 \pm 0.2$ & $24.8 \pm 0.1$ & $71.2 \pm 0.1$ & $>1000$ \\
\hline Q12 & $4-\mathrm{CH}_{3} \mathrm{OC}_{6} \mathrm{H}_{4}$ & $>1000$ & $>100$ & $64.0 \pm 0.1$ & $>1000$ \\
\hline Q13 & $2-\mathrm{NO}_{2} \mathrm{C}_{6} \mathrm{H}_{4}$ & $414.3 \pm 0.1$ & $29.9 \pm 0.1$ & $144.4 \pm 0.2$ & $>1000$ \\
\hline Q14 & $3-\mathrm{NO}_{2} \mathrm{C}_{6} \mathrm{H}_{4}$ & $298.2 \pm 0.2$ & $26.9 \pm 0.1$ & $52.6 \pm 0.1$ & $455.8 \pm 0.3$ \\
\hline Q15 & $4-\mathrm{NO}_{2} \mathrm{C}_{6} \mathrm{H}_{4}$ & $283.4 \pm 0.1$ & $31.1 \pm 0.1$ & $80.9 \pm 0.1$ & $>1000$ \\
\hline Q16 & 2- $\mathrm{HOC}_{6} \mathrm{H}_{4}$ & $458.5 \pm 0.1$ & $30.2 \pm 0.1$ & $41.7 \pm 0.1$ & $154.9 \pm 0.1$ \\
\hline Q17 & $3-\mathrm{HOC}_{6} \mathrm{H}_{4}$ & $335.9 \pm 0.1$ & $24.2 \pm 0.1$ & $44.9 \pm 0.1$ & $323.4 \pm 0.2$ \\
\hline Q18 & $4-\mathrm{HOC}_{6} \mathrm{H}_{4}$ & $822.2 \pm 0.4$ & $43.9 \pm 0.1$ & $>200$ & $372.41 \pm 0.2$ \\
\hline Q19 & 4- $\mathrm{CNC}_{6} \mathrm{H}_{4}$ & $176.0 \pm 0.1$ & $32.0 \pm 0.1$ & $>200$ & $>1000$ \\
\hline Q20 & $4-\mathrm{C}_{6} \mathrm{H}_{5} \mathrm{C}_{6} \mathrm{H}_{4}$ & $>1000$ & $43.8 \pm 0.1$ & $20.7 \pm 0.1$ & $>1000$ \\
\hline Q21 & $2-\mathrm{C}_{10} \mathrm{H}_{7}$ & $437.6 \pm 0.1$ & $43.1 \pm 0.1$ & $61.8 \pm 0.1$ & $>1000$ \\
\hline Q22 & $\mathrm{C}_{6} \mathrm{H}_{5}$ & $474.8 \pm 0.2$ & $28.8 \pm 0.1$ & $111.0 \pm 0.1$ & $>1000$ \\
\hline Trolox & & $320.2 \pm 0.1$ & $65.5 \pm 0.1$ & 139.9 & - \\
\hline Kojic acid & & - & - & - & $218.6 \pm 0.1$ \\
\hline
\end{tabular}




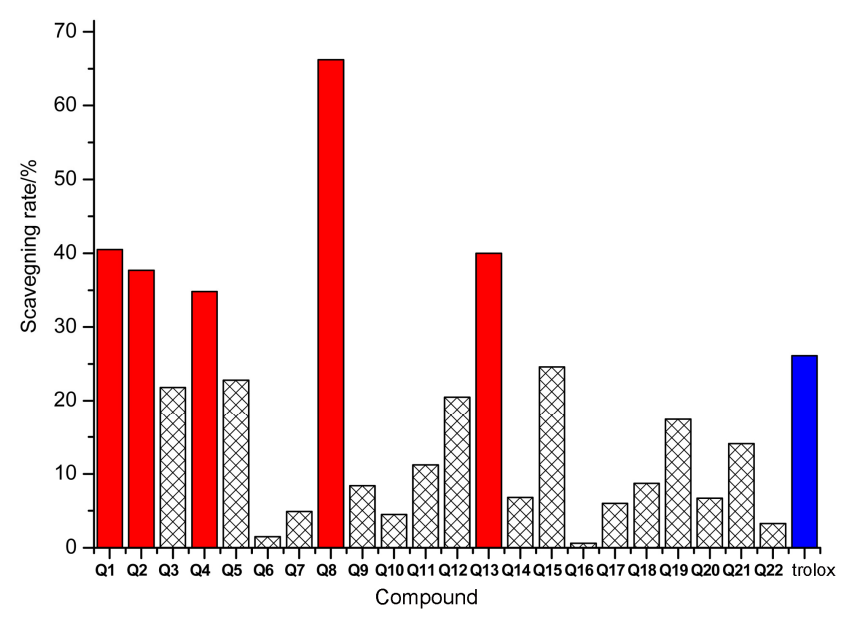

图 1 化合物 $\mathrm{Q1} \sim \mathrm{Q22}$ 与 trolox 对羟基自由基的清除效果 Figure1 Scavenging activity of compounds $\mathbf{Q 1} \sim \mathbf{Q 2 2}$ and trolox on hydroxyl free radical

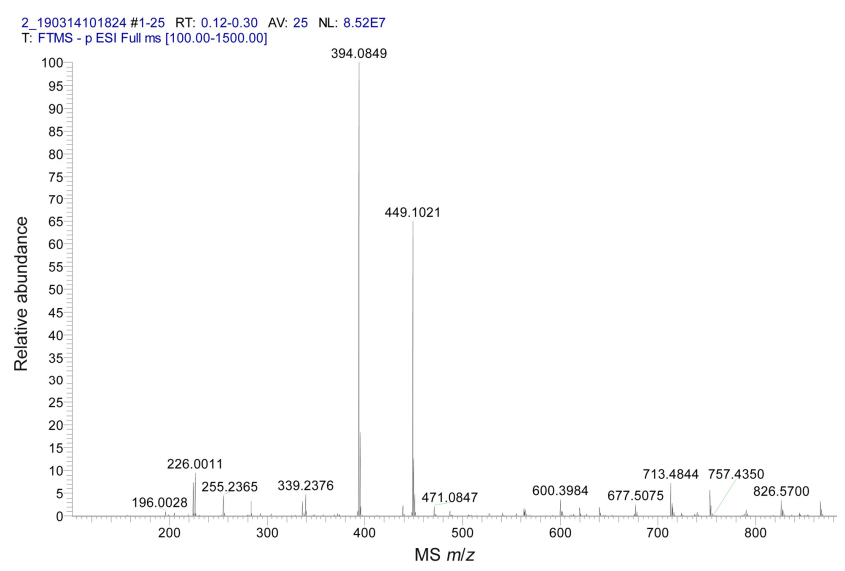

图 2 化合物 Q15 与 DPPH 自由基作用后的质谱图

Figure 2 Mass spectra of the reaction of compound Q15 with DPPH free radical

\section{4 抗氧化和抗衰老的作用机理分析}

众所周知, 维生素 $\mathrm{E}$ 是最强的抗氧化剂之一, 将 $\operatorname{Trolox}($ 水溶性维生素 E) 作为抗氧化实验的阳性对照以 准确评估樟脑磺酸噻唑腙类化合物的抗氧化活性. 采用 常见的着自由基、DPPH 自由基、ABTS 自由基和超氧
阴离子自由基体系研究了樟脑磺酸噻唑腙的抗氧化活 性. 系统中这几种自由基的浓度远高于体内浓度，此类 噻唑腙化合物可以在低药物浓度下显示出显著的抗氧 化活性, 表明此类化合物是良好的自由基清除剂及抗氧 化剂.

樟脑磺酸噻唑腙类化合物的抗氧化抗衰老机制可 分为以下四种类型: (1)直接清除活性氧. 本文通过大量 的实验表明了噻唑腙类化合物对 DPPH 自由基有较好的 清除作用，且其抗氧化能力与化合物的量显著正相关， 结合 DPPH 清除产物的质谱图, 可以推测其作用机理可 能为噻唑腙类物质通过质子转移直接清除自由基. (2)通过络合作用清除活性氧和自由基形成过程中所需 的金属离子. 噻唑腙类化合物可以与产生羟基所必须的 金属离子(如 $\mathrm{Fe}^{2+}, \mathrm{Cu}^{2+}$ ) 络合. 因此不会产生可引发脂 质过氧化的着基自由基，从而抑制活性氧物质的产生. (3)减少氧化应激. 氧化应激源于细胞不能中和过量的 氧化物质, 在过氧自由基清除实验(PSC)实验中发现此 类化合物可以将脂质过氧自由基氧化为脂质过氧化氢, 从而减少过氧自由基对细胞产生的氧化应激作用. (4)抑 制酪氨酸酶. 酪氨酸酶是黑色素形成的关键酶, 众所周 知, 黑色素可以保护皮肤免受紫外线伤害, 但是过量产 生则会氧化大脑中的多巴胺, 产生帕金森综合征相关的 神经变性. 化合物 Q16表现出了极低的酪氨酸酶半抑制 浓度，即对酪氨酸酶的高抑制活性.

\section{2 结论}

以樟脑磺酸为原料, 合成出了 22 个樟脑磺酸基噻 唑腙类化合物, 并且采用 ${ }^{1} \mathrm{H} N M R,{ }^{13} \mathrm{C} N M R$ 和 HRMS 对合成的化合物进行了结构确认. 结果表明，与阳性对 照 trolox 以及曲酸相比，化合物在很多方面均表现出优 良的的抗氧化活性. 从化合物的结构与活性关系看, 不 同的取代基会产生不同的抗氧化活性. 综上所述，此类 化合物在抗氧化抗衰老药物制备方面具有较高的开发 潜力.

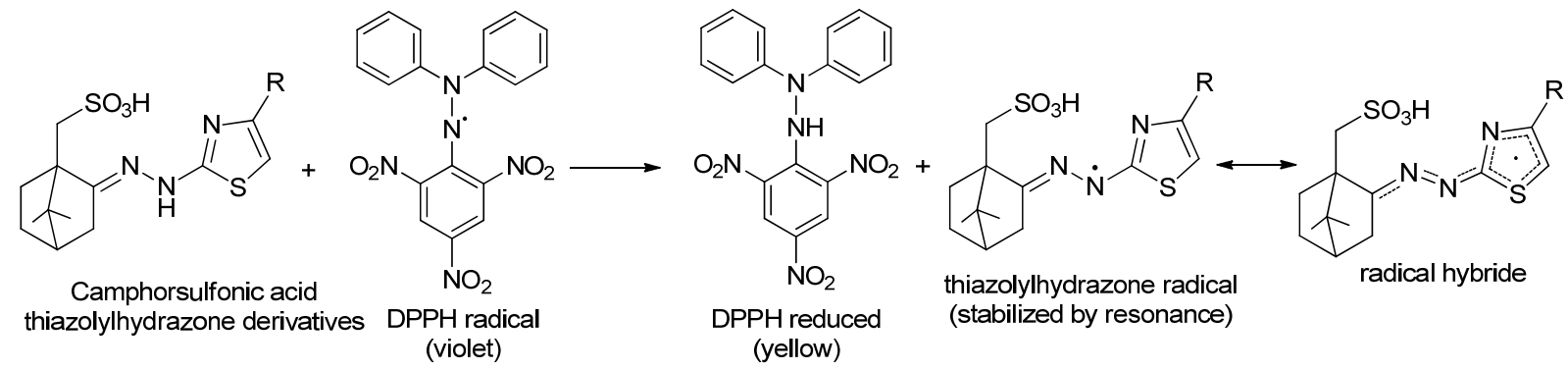

图式 2 樟脑磺酸噻唑腙类衍生物与 DPPH 自由基之间反应的示意图

Scheme 2 Schematic representation of the reaction between DPPH radical and camphorsulfonic acid thiazolylhydrazone derivatives 


\section{3 实验部分}

\section{1 仪器与试剂}

樟脑磺酸(中国国药集团); 差自由基测试试剂盒(南 京建成生物工程研究所); DPPH(购自 Sigma-Aldrich 西 格玛奥德里奇(上海)贸易有限公司), $\mathrm{ABTS}$ (购自上海阿 拉丁生化科技股份有限公司); DCFH-DA (2', $7^{\prime}$-二氯荧光 素二乙酸酯, 购自 Sigma-Aldrich 西格玛奥德里奇(上海) 贸易有限公司); ABAP (2,2'-偶氮二异丁基脒二盐酸盐, 购自上海麦克林生化科技有限公司); 其他试剂均为市 售分析纯.

DF-101S 型恒温磁力搅拌器, 南京科尔公司; 美国 Agilent 5975c 质谱仪; 德国 Bruker AV 500 核磁共振仪 (TMS 为内标, $\mathrm{CDCl}_{3}$ 或 $\mathrm{C}_{5} \mathrm{D}_{5} \mathrm{~N}$ 为溶剂); Optimelt MPA100 全自动熔点仪; UV-2450 紫外分光光度计 (日本 Shimadzu); 美国 Agilent 6540 高分辨质谱仪.

\section{2 (E)-(2-(2-氨基甲酰硫基亚肼基)-7,7-二甲基双环 [2.2.1]庚-1-基)甲磺酸(2)的合成}

将樟脑磺酸 $(1,50 \mathrm{mmol}$ )和氨基硫脲(55 mmol)加入 到 $100 \mathrm{~mL}$ 无水乙醇中, 搅拌均匀并使其充分溶解, 升 温回流反应 $1 \mathrm{~h}$. 反应完成后, 冷却过滤粉末, 并用 15 $\mathrm{mL}$ 石油醚洗涤，得到白色粉末状化合物 2, 产率 $82 \%$. m.p. $242.1 \sim 242.8{ }^{\circ} \mathrm{C} ;{ }^{1} \mathrm{H}$ NMR (400 MHz, Pyridine- $d_{5}$ ) $\delta: 10.68$ (s, 1H), 9.26 (d, $J=30.0 \mathrm{~Hz}, 2 \mathrm{H}), 8.73(\mathrm{~s}, 1 \mathrm{H})$, $3.94(\mathrm{~d}, J=14.3 \mathrm{~Hz}, 1 \mathrm{H}), 3.46(\mathrm{~d}, J=14.4 \mathrm{~Hz}, 1 \mathrm{H}), 2.81$ (t, $J=12.5 \mathrm{~Hz}, 1 \mathrm{H}), 2.60(\mathrm{dt}, J=17.2,3.2 \mathrm{~Hz}, 1 \mathrm{H}), 2.27$ (ddd, $J=13.3,9.8,3.8 \mathrm{~Hz}, 1 \mathrm{H}), 2.02(\mathrm{~d}, J=17.3 \mathrm{~Hz}$, 1H), 1.79 (s, 2H), 1.16 (s, 1H), 0.98 (s, 3H), 0.73 (s, 3H); ${ }^{13} \mathrm{C}$ NMR (101 MHz, Pyridine- $d_{5}$ ) $\delta$ : 181.52, 163.07, $55.25,52.06,49.85,44.57,34.74,29.42,27.89,19.89$, 19.54; HRMS calcd for $\mathrm{C}_{11} \mathrm{H}_{20} \mathrm{~N}_{3} \mathrm{O}_{3} \mathrm{~S}_{2}[\mathrm{M}+\mathrm{H}]^{+}$306.0951, found 306.0946 .

\section{3 化合物 $\mathrm{Q} 1 \sim \mathrm{Q} 22$ 的合成}

将 $3 \mathrm{mmol}$ 化合物 2 置于 $25 \mathrm{~mL}$ 三口烧瓶中, 加入 $10 \mathrm{~mL}$ 乙醇和 $1.5 \mathrm{~mL}$ 超纯水, 室温下搅拌使其充分溶 解, 然后加入 $3.3 \mathrm{mmol}$ 的溴代苯乙酮, 反应液逐渐变成 浑浊并形成悬浮液后停止反应, 抽滤, 滤饼经石油醚洗 涤 3 次，烘干，得到目标化合物 Q1 $\sim \mathbf{Q 2 2}$.

(2-(2-(4-(2-氟苯基)噻唑-2-基)亚肼基)-7,7-二甲基双 环[2.2.1]庚-1-基)甲磺酸(Q1)：产率 88\%. m. p. 264.7 $265.1{ }^{\circ} \mathrm{C} ;{ }^{1} \mathrm{H}$ NMR (400 MHz, DMSO- $\left.d_{6}\right) \delta: 7.96$ (t, $J=$ $7.3 \mathrm{~Hz}, 1 \mathrm{H}), 7.44 \sim 7.35(\mathrm{~m}, 1 \mathrm{H}), 7.34 \sim 7.23(\mathrm{~m}, 2 \mathrm{H}), 7.19$ (s, 1H), 3.44 (q, $J=7.0 \mathrm{~Hz}, 1 \mathrm{H}), 3.26(\mathrm{~d}, J=14.9 \mathrm{~Hz}, 1 \mathrm{H})$, $2.72(\mathrm{~d}, J=14.9 \mathrm{~Hz}, 1 \mathrm{H}), 2.57$ (d, $J=11.6 \mathrm{~Hz}, 1 \mathrm{H}), 2.07$ (d, $J=18.0 \mathrm{~Hz}, 1 \mathrm{H}), 1.91(\mathrm{t}, J=4.0 \mathrm{~Hz}, 1 \mathrm{H}), 1.82(\mathrm{~s}, 1 \mathrm{H})$, $1.49(\mathrm{~s}, 1 \mathrm{H}), 1.27 \sim 1.16(\mathrm{~m}, 1 \mathrm{H}), 1.05(\mathrm{~s}, 3 \mathrm{H}), 0.75(\mathrm{~s}$, $3 \mathrm{H}) ;{ }^{13} \mathrm{C}$ NMR $\left(100 \mathrm{MHz}\right.$, Pyridine- $\left.d_{5}\right) \delta: 170.60,164.33$, $161.02\left(J_{\mathrm{C}-\mathrm{F}}=247.95 \mathrm{~Hz}\right), 145.76,130.55,130.52,129.22$ $\left(J_{\mathrm{C}-\mathrm{F}}=8.43 \mathrm{~Hz}\right), 116.53\left(J_{\mathrm{C}-\mathrm{F}}=22.4 \mathrm{~Hz}\right), 108.86,108.70$, $54.66,50.55,49.60,45.13,35.62,28.95,28.01,20.78$, 20.02; HRMS calcd for $\mathrm{C}_{19} \mathrm{H}_{23} \mathrm{FN}_{3} \mathrm{O}_{3} \mathrm{~S}_{2}[\mathrm{M}+\mathrm{H}]{ }^{+}$ 424.1163 , found 424.1165 .

(2-(2-(4-(3-氟苯基)噻唑-2-基)亚肼基)-7,7-二甲基双 环[2.2.1]庚-1-基)甲磺酸(Q2): 产率 94.8\%. m.p. 283.3 $283.8{ }^{\circ} \mathrm{C} ;{ }^{1} \mathrm{H}$ NMR $\left(400 \mathrm{MHz}\right.$, DMSO- $\left.d_{6}\right) \delta$ : 7.67 (t, $J=$ $9.6 \mathrm{~Hz}, 2 \mathrm{H}), 7.50 \sim 7.42(\mathrm{~m}, 1 \mathrm{H}), 7.40(\mathrm{~s}, 1 \mathrm{H}), 7.16$ (t, $J=$ $8.4 \mathrm{~Hz}, 1 \mathrm{H}), 3.25(\mathrm{~d}, J=14.8 \mathrm{~Hz}, 1 \mathrm{H}), 2.72(\mathrm{~d}, J=14.8$ $\mathrm{Hz}, 1 \mathrm{H}), 2.58(\mathrm{~d}, J=13.8 \mathrm{~Hz}, 1 \mathrm{H}), 2.45(\mathrm{~s}, 1 \mathrm{H}), 2.07(\mathrm{~d}$, $J=18.0 \mathrm{~Hz}, 1 \mathrm{H}), 1.92(\mathrm{~s}, 1 \mathrm{H}), 1.83(\mathrm{~s}, 1 \mathrm{H}), 1.53(\mathrm{~s}, 1 \mathrm{H})$, $1.22(\mathrm{~s}, 1 \mathrm{H}), 1.04(\mathrm{~s}, 3 \mathrm{H}), 0.75(\mathrm{~s}, 3 \mathrm{H}) ;{ }^{13} \mathrm{C}$ NMR $(100$ $\mathrm{MHz}$, Pyridine- $\left.d_{5}\right) \delta: 171.53,165.12,164.35,162.70$, $138.83\left(J_{\mathrm{C}-\mathrm{F}}=8.05 \mathrm{~Hz}\right), 130.92\left(J_{\mathrm{C}-\mathrm{F}}=8.25 \mathrm{~Hz}\right), 122.34$, $114.53\left(J_{\mathrm{C}-\mathrm{F}}=21.16 \mathrm{~Hz}\right), 113.44\left(J_{\mathrm{C}-\mathrm{F}}=22.76 \mathrm{~Hz}\right), 105.14$, $54.69,50.57,49.63,45.15,35.64,28.96,28.03,20.80$, 20.05; HRMS calcd for $\mathrm{C}_{19} \mathrm{H}_{23} \mathrm{FN}_{3} \mathrm{O}_{3} \mathrm{~S}_{2}[\mathrm{M}+\mathrm{H}]$ 424.1156 , found 424.1165 .

(2-(2-(4-(4-氟苯基)噻唑-2-基)亚肼基)-7,7-二甲基双 环[2.2.1]庚-1-基)甲磺酸(Q3): 产率 90.2\%. m.p. 288.7 $289.4{ }^{\circ} \mathrm{C}$. ${ }^{1} \mathrm{H}$ NMR $\left(400 \mathrm{MHz}\right.$, Pyridine- $\left.d_{5}\right) \delta$ : 10.21 (s, $1 \mathrm{H}), 9.52(\mathrm{dd}, J=8.6,5.6 \mathrm{~Hz}, 2 \mathrm{H}), 9.06(\mathrm{~s}, 1 \mathrm{H}), 8.57$ (s, $1 \mathrm{H}), 5.83(\mathrm{~d}, J=15.0 \mathrm{~Hz}, 1 \mathrm{H}), 5.14(\mathrm{~d}, J=15.0 \mathrm{~Hz}, 1 \mathrm{H})$, $4.96(\mathrm{t}, J=10.2 \mathrm{~Hz}, 1 \mathrm{H}), 4.24(\mathrm{~d}, J=17.0 \mathrm{~Hz}, 1 \mathrm{H}), 3.77$ (d, $J=17.0 \mathrm{~Hz}, 1 \mathrm{H}), 3.55 \sim 3.41(\mathrm{~m}, 2 \mathrm{H}), 3.39(\mathrm{~s}, 1 \mathrm{H})$, 2.94 (s, 3H), $2.79 \sim 2.67(\mathrm{~m}, 1 \mathrm{H}), 2.42(\mathrm{~s}, 3 \mathrm{H}) ;{ }^{13} \mathrm{C} \mathrm{NMR}$ $\left(100 \mathrm{MHz}\right.$, Pyridine- $\left.d_{5}\right) \delta: 173.20,165.88,163.25,152.53$, $130.07\left(J_{\mathrm{C}-\mathrm{F}}=7.87 \mathrm{~Hz}\right), 134.51\left(J_{\mathrm{C}-\mathrm{F}}=3.15 \mathrm{~Hz}\right), 117.60$ $\left(J_{\mathrm{C}-\mathrm{F}}=21.37 \mathrm{~Hz}\right), 105.10,56.30,52.20,51.25,46.76$, 37.26, 30.58, 29.65, 22.43, 21.66; HRMS calcd for $\mathrm{C}_{19} \mathrm{H}_{23^{-}}$ $\mathrm{FN}_{3} \mathrm{O}_{3} \mathrm{~S}_{2}[\mathrm{M}+\mathrm{H}]^{+}$424.1157, found 424.1165 .

(2-(2-(4-(2-氯苯基)噻唑-2-基)亚肼基)-7,7-二甲基双 环[2.2.1]庚-1-基)甲磺酸(Q4): 产率 91.2\%. m.p. 252.5 $253.2{ }^{\circ} \mathrm{C} ;{ }^{1} \mathrm{H}$ NMR $(400 \mathrm{MHz}$, Chloroform- $d$ ) $\delta: 7.58(\mathrm{~d}$, $J=7.1 \mathrm{~Hz}, 1 \mathrm{H}), 7.46(\mathrm{~d}, J=7.4 \mathrm{~Hz}, 1 \mathrm{H}), 7.41 \sim 7.28(\mathrm{~m}$, $2 \mathrm{H}), 6.81(\mathrm{~s}, 1 \mathrm{H}), 3.71(\mathrm{q}, J=7.0 \mathrm{~Hz}, 1 \mathrm{H}), 3.54(\mathrm{~d}, J=$ $14.8 \mathrm{~Hz}, 1 \mathrm{H}), 3.02(\mathrm{~d}, J=14.7 \mathrm{~Hz}, 1 \mathrm{H}), 2.17$ (d, $J=18.3$ $\mathrm{Hz}, 1 \mathrm{H}), 1.96(\mathrm{~s}, 1 \mathrm{H}), 1.86(\mathrm{~s}, 1 \mathrm{H}), 1.71(\mathrm{~s}, 1 \mathrm{H}), 1.23(\mathrm{t}$, $J=7.0 \mathrm{~Hz}, 2 \mathrm{H}), 0.99$ (s, 3H), 0.76 (s, 3H); ${ }^{13} \mathrm{C}$ NMR $(100$ $\mathrm{MHz}$, Pyridine- $\left.d_{5}\right) \delta: 170.46,164.29,148.47,134.91$, $132.26,132.16,131.19,129.08,127.57,109.13,54.68$, $50.59,49.61,45.16,35.65,28.99,28.04,20.79,20.05$; 
HRMS calcd for $\mathrm{C}_{19} \mathrm{H}_{23} \mathrm{ClN}_{3} \mathrm{O}_{3} \mathrm{~S}_{2}[\mathrm{M}+\mathrm{H}]^{+}$440.0866, found 440.0869 .

(2-(2-(4-(3-氯苯基)噻唑-2-基)亚肼基)-7,7-二甲基双 环[2.2.1]庚-1-基)甲磺酸(Q5): 产率 85\%. m.p. 293.8 $294.4{ }^{\circ} \mathrm{C} ;{ }^{1} \mathrm{H}$ NMR (400 MHz, DMSO- $\left.d_{6}\right) \delta$ : $7.90(\mathrm{~s}, 1 \mathrm{H})$, $7.79(\mathrm{~d}, J=7.3 \mathrm{~Hz}, 1 \mathrm{H}), 7.49 \sim 7.40(\mathrm{~m}, 2 \mathrm{H}), 7.38$ (d, $J=$ $7.9 \mathrm{~Hz}, 1 \mathrm{H}), 3.25$ (d, $J=14.8 \mathrm{~Hz}, 1 \mathrm{H}), 2.71$ (d, $J=14.9$ $\mathrm{Hz}, 1 \mathrm{H}), 2.59$ (d, $J=15 \mathrm{~Hz}, 1 \mathrm{H}), 2.06$ (d, $J=17.9 \mathrm{~Hz}, 1 \mathrm{H})$, $1.91(\mathrm{~s}, 1 \mathrm{H}), 1.83(\mathrm{~s}, 1 \mathrm{H}), 1.50(\mathrm{~s}, 1 \mathrm{H}), 1.21(\mathrm{~s}, 1 \mathrm{H}), 1.05$ $(\mathrm{s}, 3 \mathrm{H}), 0.75(\mathrm{~s}, 3 \mathrm{H}) ;{ }^{13} \mathrm{C}$ NMR (100 MHz, Pyridine- $\left.d_{5}\right) \delta$ : $171.64,164.44,149.74,138.45,135.09,130.84,127.83$, 126.93, 124.84, 105.27, 54.81, 50.68, 49.76, 45.28, 35.76, $29.08,28.15,20.93,20.17$; HRMS calcd for $\mathrm{C}_{19} \mathrm{H}_{23} \mathrm{Cl}-$ $\mathrm{N}_{3} \mathrm{O}_{3} \mathrm{~S}_{2}[\mathrm{M}+\mathrm{H}]^{+}$440.0863, found 440.0869.

(2-(2-(4-(4-氯苯基)噻唑-2-基)亚肼基)-7,7-二甲基双 环[2.2.1]庚-1-基)甲磺酸(Q6): 产率 90.2\%. m.p. 293.9 $294.2{ }^{\circ} \mathrm{C} ;{ }^{1} \mathrm{H}$ NMR (400 MHz, DMSO-d6) $\delta: 7.85$ (d, $J=$ $8.3 \mathrm{~Hz}, 2 \mathrm{H}), 7.47$ (d, $J=8.2 \mathrm{~Hz}, 2 \mathrm{H}), 7.33$ (s, 1H), 3.24 (d, $J=14.8 \mathrm{~Hz}, 1 \mathrm{H}), 2.73(\mathrm{~d}, J=14.8 \mathrm{~Hz}, 1 \mathrm{H}), 2.07$ (d, $J=$ $17.6 \mathrm{~Hz}, 1 \mathrm{H}), 1.92$ (s, 1H), 1.83 (s, 1H), 1.53 (s, 1H), 1.22 (s, 1H), $1.17(\mathrm{~s}, 1 \mathrm{H}), 1.04(\mathrm{~s}, 3 \mathrm{H}), 0.75(\mathrm{~s}, 3 \mathrm{H}) ;{ }^{13} \mathrm{C} \mathrm{NMR}$ $\left(100 \mathrm{MHz}\right.$, Pyridine- $\left.d_{5}\right) \delta: 173.21,165.96,152.37,136.73$, 134.86, 130.93, 129.72, 106.09, 56.31, 52.19, 51.25, 46.77, $37.26,30.59$, 29.65, 22.43, 21.67; HRMS calcd for $\mathrm{C}_{19} \mathrm{H}_{23} \mathrm{ClN}_{3} \mathrm{O}_{3} \mathrm{~S}_{2}[\mathrm{M}+\mathrm{H}]^{+}$440.0861, found 440.0869.

(2-(2-(4-(2-甲基苯基)噻唑-2-基)亚肼基)-7,7-二甲基 双环[2.2.1]庚-1-基)甲磺酸(Q7)：产率 86\%. m.p. 281.4 $282.0{ }^{\circ} \mathrm{C} ;{ }^{1} \mathrm{H}$ NMR (400 MHz, DMSO- $\left.d_{6}\right) \delta: 7.43$ (d, $J=$ $7.5 \mathrm{~Hz}, 1 \mathrm{H}), 7.34$ (dd, $J=13.4,6.3 \mathrm{~Hz}, 2 \mathrm{H}), 7.28$ (q, $J=$ $7.6 \mathrm{~Hz}, 1 \mathrm{H}), 6.95$ (s, 1H), 3.22 (d, J=14.7 Hz, 1H), 2.72 (d, $J=14.7 \mathrm{~Hz}, 1 \mathrm{H}), 2.37$ (s, 3H), 2.08 (d, $J=17.9 \mathrm{~Hz}$, 1H), 1.93 (s, 1H), 1.82 (s, 1H), 1.57 (s, 1H), $1.22(\mathrm{~s}, 1 \mathrm{H})$, $1.00(\mathrm{~s}, 3 \mathrm{H}), 0.75(\mathrm{~s}, 3 \mathrm{H}) ;{ }^{13} \mathrm{C}$ NMR $(100 \mathrm{MHz}$, Pyridine- $\left.d_{5}\right) \delta: 171.93,165.46,153.53,137.86,137.72,132.84$, $131.58,129.45,127.74,107.89,55.93,51.87,50.85,46.41$, 36.91, 30.27, 29.30, 23.13, 22.05, 21.30; HRMS calcd for $\mathrm{C}_{20} \mathrm{H}_{26} \mathrm{~N}_{3} \mathrm{O}_{3} \mathrm{~S}_{2}[\mathrm{M}+\mathrm{H}]^{+}$420.1409, found 420.1415.

(2-(2-(4-(3-甲基苯基)噻唑-2-基)亚肼基)-7,7-二甲基 双环 [2.2.1] 庚-1-基) 甲磺酸 (Q8): 产率 80.5\%. m.p. $289.7 \sim 290.3{ }^{\circ} \mathrm{C} ;{ }^{1} \mathrm{H}$ NMR (400 MHz, Pyridine- $\left.d_{5}\right) \delta$ : $7.94(\mathrm{~d}, J=6.3 \mathrm{~Hz}, 2 \mathrm{H}), 7.34(\mathrm{t}, J=7.9 \mathrm{~Hz}, 1 \mathrm{H}), 7.15 \sim$ $7.08(\mathrm{~m}, 2 \mathrm{H}), 4.38$ (d, $J=14.9 \mathrm{~Hz}, 1 \mathrm{H}), 3.69$ (d, $J=15.0$ $\mathrm{Hz}, 1 \mathrm{H}), 3.52$ (d, $J=12.8 \mathrm{~Hz}, 1 \mathrm{H}), 2.78$ (dt, $J=17.0,3.9$ $\mathrm{Hz}, 1 \mathrm{H}), 2.32$ (s, 1H), $2.28(\mathrm{~d}, J=3.5 \mathrm{~Hz}, 3 \mathrm{H}), 2.09 \sim 1.94$ (m, 2H), 1.93 (d, J=4.2 Hz, 1H), 1.49 (s, 3H), 1.26 (ddd,
$J=12.7,9.0,4.0 \mathrm{~Hz}, 1 \mathrm{H}), 0.96(\mathrm{~s}, 3 \mathrm{H}) ;{ }^{13} \mathrm{C} \mathrm{NMR}(101$ $\mathrm{MHz}$, Pyridine- $\left.d_{5}\right) \delta: 171.46,164.14,152.29,148.68$, $138.64,129.32,128.90,127.64,103.68,54.78,50.72$, 49.74, 45.30, 35.73, 29.11, 28.17, 21.89, 20.95, 20.17; HRMS $\left(\mathrm{ESI}^{+}\right.$) calcd for $\mathrm{C}_{20} \mathrm{H}_{26} \mathrm{~N}_{3} \mathrm{O}_{3} \mathrm{~S}_{2}[\mathrm{M}+\mathrm{H}]^{+}$420.1394, found 420.1415 .

(2-(2-(4-(4-甲基苯基)噻唑-2-基)亚肼基)-7,7-二甲基 双环[2.2.1]庚-1-基)甲磺酸(Q9)：产率 87\%. m.p. 241.5 $242.0{ }^{\circ} \mathrm{C}$; ${ }^{1} \mathrm{H}$ NMR (400 MHz, Pyridine- $\left.d_{5}\right) \delta: 8.03$ (d, $J=8.1 \mathrm{~Hz}, 2 \mathrm{H}), 7.59(\mathrm{~s}, 1 \mathrm{H}), 7.20(\mathrm{~s}, 1 \mathrm{H}), 7.10(\mathrm{~s}, 1 \mathrm{H})$, 4.37 (d, $J=15.0 \mathrm{~Hz}, 1 \mathrm{H}), 3.68$ (d, $J=15.0 \mathrm{~Hz}, 1 \mathrm{H}), 3.49$ (td, $J=12.7,12.2,4.2 \mathrm{~Hz}, 1 \mathrm{H}), 2.77$ (d, $J=16.9 \mathrm{~Hz}, 1 \mathrm{H})$, $2.30(\mathrm{~d}, J=17.0 \mathrm{~Hz}, 1 \mathrm{H}), 2.24(\mathrm{~s}, 3 \mathrm{H}), 2.09 \sim 1.94(\mathrm{~m}$, 2H), 1.92 (s, 1H), 1.48 (s, 3H), $1.26(\mathrm{td}, J=10.4,9.0,4.0$ $\mathrm{Hz}, 1 \mathrm{H}), 0.95$ (s, 3H); ${ }^{13} \mathrm{C}$ NMR (100 MHz, Pyridine- $\left.d_{5}\right) \delta$ : $171.40,164.17,152.06,137.62$, 133.80, 129.97, 126.69, 102.91, 54.69, 50.62, 49.64, 45.18, 35.64, 29.00, 28.06, 21.46, 20.84, 20.07; HRMS calcd for $\mathrm{C}_{20} \mathrm{H}_{26} \mathrm{~N}_{3} \mathrm{O}_{3} \mathrm{~S}_{2}[\mathrm{M}+$ $\mathrm{H}]^{+}$420.1394, found 420.1415 .

(2-(2-(4-(2-甲氧基苯基)噻唑-2-基)亚肼基)-7,7-二甲 基双环[2.2.1]庚-1-基) 甲磺酸(Q10): 产率 89\%. m.p. 276.6 277.0 ${ }^{\circ} \mathrm{C} ;{ }^{1} \mathrm{H}$ NMR (400 MHz, DMSO-d 6 ) $\delta: 7.79$ (d, $J=7.7 \mathrm{~Hz}, 1 \mathrm{H}), 7.39$ (t, $J=7.6 \mathrm{~Hz}, 1 \mathrm{H}), 7.27(\mathrm{~s}, 1 \mathrm{H})$, $7.14(\mathrm{~d}, J=8.3 \mathrm{~Hz}, 1 \mathrm{H}), 7.01(\mathrm{t}, J=7.5 \mathrm{~Hz}, 1 \mathrm{H}), 3.89$ (s, $3 \mathrm{H}), 3.22(\mathrm{~d}, J=14.7 \mathrm{~Hz}, 1 \mathrm{H}), 2.72(\mathrm{~d}, J=14.7 \mathrm{~Hz}, 1 \mathrm{H})$, 2.54 (s, 1H), 2.09 (d, J=18.0 Hz, 1H), 1.93 (s, 1H), 1.83 $(\mathrm{s}, 1 \mathrm{H}), 1.57(\mathrm{~s}, 1 \mathrm{H}), 1.22(\mathrm{t}, J=10.6 \mathrm{~Hz}, 1 \mathrm{H}), 1.02(\mathrm{~s}$, $3 \mathrm{H}), 0.76(\mathrm{~s}, 3 \mathrm{H}) ;{ }^{13} \mathrm{C} \mathrm{NMR}\left(100 \mathrm{MHz}\right.$, Pyridine- $\left.d_{5}\right) \delta$ : $171.36,164.24,160.03,151.66,135.39,129.29,128.00$, $123.31,114.79,101.78,55.58,54.67,50.59,49.62,45.14$, $35.61,28.97,28.03,20.80,20.04$; HRMS calcd for $\mathrm{C}_{20} \mathrm{H}_{26} \mathrm{~N}_{3} \mathrm{O}_{4} \mathrm{~S}_{2}[\mathrm{M}+\mathrm{H}]^{+}$436.1357, found 436.1364.

(2-(2-(4-(3-甲氧基苯基)噻唑-2-基)亚肼基)-7,7-二甲 基双环[2.2.1]庚-1-基)甲磺酸(Q11)：产率 91\%. m.p. 282.3 283.2 ${ }^{\circ} \mathrm{C} ;{ }^{1} \mathrm{H}$ NMR (400 MHz, DMSO-d 6 ) $\delta: 7.44$ (s, 1H), 7.40 (d, $J=7.8 \mathrm{~Hz}, 1 \mathrm{H}), 7.35$ (d, $J=2.4 \mathrm{~Hz}, 1 \mathrm{H})$, 7.32 (d, $J=7.9 \mathrm{~Hz}, 1 \mathrm{H}), 6.94(\mathrm{dd}, J=8.1,1.7 \mathrm{~Hz}, 1 \mathrm{H})$, 3.82 (s, 3H), 3.25 (d, $J=14.7 \mathrm{~Hz}, 1 \mathrm{H}), 2.77$ (d, $J=14.7$ $\mathrm{Hz}, 1 \mathrm{H}), 2.55$ (s, 1H), 2.45 (d, $J=11.6 \mathrm{~Hz}, 1 \mathrm{H}), 2.10$ (d, $J=17.9 \mathrm{~Hz}, 1 \mathrm{H}), 1.95(\mathrm{t}, J=4.0 \mathrm{~Hz}, 1 \mathrm{H}), 1.90 \sim 1.76(\mathrm{~m}$, $1 \mathrm{H}), 1.64(\mathrm{~s}, 1 \mathrm{H}), 1.24(\mathrm{~s}, 1 \mathrm{H}), 1.02(\mathrm{~s}, 3 \mathrm{H}), 0.77(\mathrm{~s}, 3 \mathrm{H})$; ${ }^{13} \mathrm{C}$ NMR (100 MHz, Pyridine- $\left.d_{5}\right) \delta: 172.97,165.74$, $162.47,153.54,139.51,131.92,120.69,115.64,113.94$, $105.72,57.12$, 56.30, 52.21, 51.24, 46.79, 37.25, 30.61, 29.67, 22.45, 21.67; HRMS calcd for $\mathrm{C}_{20} \mathrm{H}_{26} \mathrm{~N}_{3} \mathrm{O}_{4} \mathrm{~S}_{2}[\mathrm{M}+$ 
$\mathrm{H}]^{+}$436.1356, found 436.1364.

(2-(2-(4-(4-甲氧基苯基)噻唑-2-基)亚肼基)-7,7-二甲 基双环[2.2.1]庚-1-基) 甲磺酸(Q12): 产率 93\%. m.p. $273.7 \sim 274.2{ }^{\circ} \mathrm{C} ;{ }^{1} \mathrm{H}$ NMR (400 MHz, DMSO- $\left.d_{6}\right) \delta: 7.77$ (d, $J=8.7 \mathrm{~Hz}, 2 \mathrm{H}), 7.14$ (s, 1H), 6.98 (d, $J=8.8 \mathrm{~Hz}, 2 \mathrm{H})$, 3.79 (s, 3H), 3.23 (d, $J=14.7 \mathrm{~Hz}, 1 \mathrm{H}), 2.76$ (d, $J=14.7$ $\mathrm{Hz}, 1 \mathrm{H}), 2.55$ (s, 1H), 2.47 2.37 (m, 1H), 2.09 (d, $J=$ $18.0 \mathrm{~Hz}, 1 \mathrm{H}), 1.94$ (s, 1H), 1.83 (s, 1H), 1.64 (s, 1H), 1.23 $(\mathrm{s}, 1 \mathrm{H}), 1.01(\mathrm{~s}, 3 \mathrm{H}), 0.76(\mathrm{~s}, 3 \mathrm{H}) ;{ }^{13} \mathrm{C} \mathrm{NMR}(100 \mathrm{MHz}$, Pyridine- $\left.d_{5}\right) \delta$ : 169.68, 164.14, 157.97, 147.85, 130.59, 129.03, 124.91, 121.42, 112.16, 108.66, 55.78, 54.76, 50.73, 49.72, 45.29, 35.7017, 29.14, 28.16, 20.97, 20.17; HRMS calcd for $\mathrm{C}_{20} \mathrm{H}_{26} \mathrm{~N}_{3} \mathrm{O}_{4} \mathrm{~S}_{2}[\mathrm{M}+\mathrm{H}]^{+}$436.1362, found 436.1364

(2-(2-(4-(2-硝基苯基)噻唑-2-基)亚肼基)-7,7-二甲基 双环 [2.2.1] 庚-1-基) 甲磺酸 (Q13): 产率 86\%. m.p. 251.6 252.0 ${ }^{\circ} \mathrm{C} ;{ }^{1} \mathrm{H}$ NMR (400 MHz, DMSO- $d_{6}$ ) $\delta: 7.96$ $(\mathrm{d}, J=8.1 \mathrm{~Hz}, 1 \mathrm{H}), 7.79 \sim 7.71(\mathrm{~m}, 2 \mathrm{H}), 7.64(\mathrm{ddd}, J=8.7$, 5.7, $3.2 \mathrm{~Hz}, 1 \mathrm{H}), 7.11(\mathrm{~s}, 1 \mathrm{H}), 3.27$ (d, $J=14.9 \mathrm{~Hz}, 1 \mathrm{H})$, $2.71(\mathrm{~d}, J=14.9 \mathrm{~Hz}, 1 \mathrm{H}), 2.61(\mathrm{t}, J=11.9 \mathrm{~Hz}, 1 \mathrm{H}), 2.45$ (s, $1 \mathrm{H}), 2.05(\mathrm{~d}, J=18.0 \mathrm{~Hz}, 1 \mathrm{H}), 1.89$ (t, $J=4.0 \mathrm{~Hz}, 1 \mathrm{H})$, $1.82(\mathrm{~d}, J=3.1 \mathrm{~Hz}, 1 \mathrm{H}), 1.43(\mathrm{~s}, 1 \mathrm{H}), 1.20(\mathrm{~s}, 1 \mathrm{H}), 1.06(\mathrm{~s}$, $3 \mathrm{H}), 0.75(\mathrm{~s}, 3 \mathrm{H}) ;{ }^{13} \mathrm{C}$ NMR (100 MHz, Pyridine- $\left.d_{5}\right) \delta$ : $171.89,164.43,148.23,132.50,131.35,130.56,128.98$, 124.58, 124.36, 107.83, 54.75, 50.57, 49.61, 45.14, 35.83, 28.97, 28.04, 20.76, 20.06; HRMS calcd for $\mathrm{C}_{19} \mathrm{H}_{23} \mathrm{~N}_{4} \mathrm{O}_{5} \mathrm{~S}_{2}$ $[\mathrm{M}+\mathrm{H}]^{+}$451.1103, found 451.1110.

(2-(2-(4-(3-硝基苯基)噻唑-2-基)亚肼基)-7,7-二甲基 双环 [2.2.1]庚-1-基) 甲磺酸(Q14): 产率 87.8\%. m.p. $281.1 \sim 281.5{ }^{\circ} \mathrm{C} ;{ }^{1} \mathrm{H}$ NMR (400 MHz, DMSO- $\left.d_{6}\right) \delta: 8.66$ (s, 1H), 8.27 (d, $J=7.8 \mathrm{~Hz}, 1 \mathrm{H}), 8.15(\mathrm{~d}, J=8.1 \mathrm{~Hz}, 1 \mathrm{H})$, 7.69 (t, $J=8.0 \mathrm{~Hz}, 1 \mathrm{H}), 7.58$ (s, 1H), 3.29 (d, $J=14.9 \mathrm{~Hz}$, 1H), 2.73 (d, $J=14.9 \mathrm{~Hz}, 1 \mathrm{H}), 2.64$ (t, $J=11.7 \mathrm{~Hz}, 1 \mathrm{H})$, $2.46(\mathrm{~s}, 1 \mathrm{H}), 2.06(\mathrm{~d}, J=17.9 \mathrm{~Hz}, 1 \mathrm{H}), 1.90(\mathrm{~s}, 1 \mathrm{H}), 1.83$ $(\mathrm{s}, 1 \mathrm{H}), 1.46(\mathrm{~s}, 1 \mathrm{H}), 1.21(\mathrm{~s}, 1 \mathrm{H}), 1.07(\mathrm{~s}, 3 \mathrm{H}), 0.75(\mathrm{~s}$, $3 \mathrm{H}) ;{ }^{13} \mathrm{C}$ NMR (100 MHz, Pyridine- $\left.d_{5}\right) \delta: 171.87,164.62$, $149.61,149.49$, 137.93, 132.17, 130.30, 122.42, 121.51, 106.44, 54.86, 50.68, 49.80, 45.29, 35.87, 29.09, 28.17, 20.94, 20.19; HRMS calcd for $\mathrm{C}_{19} \mathrm{H}_{23} \mathrm{~N}_{4} \mathrm{O}_{5} \mathrm{~S}_{2}[\mathrm{M}+\mathrm{H}]^{+}$ 451.1095 , found 451.1110

(2-(2-(4-(4-硝基苯基)噻唑-2-基)亚肼基)-7,7-二甲基 双环 [2.2.1] 庚-1-基) 甲磺酸(Q15): 产率 86.8\%. m.p. $291.0 \sim 291.9{ }^{\circ} \mathrm{C} ;{ }^{1} \mathrm{H}$ NMR (400 MHz, DMSO- $\left.d_{6}\right) \delta: 8.26$ $(\mathrm{d}, J=9.0 \mathrm{~Hz}, 2 \mathrm{H}), 8.10$ (d, $J=8.9 \mathrm{~Hz}, 2 \mathrm{H}), 7.65$ (s, 1H), $3.30(\mathrm{~d}, J=14.9 \mathrm{~Hz}, 1 \mathrm{H}), 2.75(\mathrm{~d}, J=14.9 \mathrm{~Hz}, 1 \mathrm{H}), 2.69 \sim$ $2.58(\mathrm{~m}, 1 \mathrm{H}), 2.47(\mathrm{~s}, 1 \mathrm{H}), 2.07(\mathrm{~d}, J=17.8 \mathrm{~Hz}, 1 \mathrm{H}), 1.91$ (t, $J=4.1 \mathrm{~Hz}, 1 \mathrm{H}), 1.83$ (dd, $J=8.0,3.9 \mathrm{~Hz}, 1 \mathrm{H}), 1.52 \sim$ $1.41(\mathrm{~m}, 1 \mathrm{H}), 1.23(\mathrm{dd}, J=8.7,3.6 \mathrm{~Hz}, 1 \mathrm{H}), 1.08$ (s, 3H), $0.76(\mathrm{~s}, 3 \mathrm{H}) ;{ }^{13} \mathrm{C}$ NMR (100 MHz, Pyridine- $\left.d_{5}\right) \delta: 171.79$, $164.67,147.15,142.11,126.96,124.57,124.32,108.54$, $54.71,50.51,49.62,45.09,35.69,28.90,27.98,20.75$, 20.01; HRMS calcd for $\mathrm{C}_{19} \mathrm{H}_{23} \mathrm{~N}_{4} \mathrm{O}_{5} \mathrm{~S}_{2}[\mathrm{M}+\mathrm{H}]^{+} 451.1102$, found 451.1110 .

(2-(2-(4-(2-着基苯基)噻唑-2-基)亚肼基)-7,7-二甲基 双环 [2.2.1] 庚-1-基) 甲磺酸 (Q16): 产率 91\%. m.p. $252.1 \sim 253.0{ }^{\circ} \mathrm{C} ;{ }^{1} \mathrm{H}$ NMR (400 MHz, Pyridine- $\left.d_{5}\right) \delta$ : $9.34(\mathrm{dd}, J=7.9,1.5 \mathrm{~Hz}, 1 \mathrm{H}), 8.93 \sim 8.87(\mathrm{~m}, 1 \mathrm{H}), 8.60$ (d, $J=1.0 \mathrm{~Hz}, 1 \mathrm{H}), 8.59 \sim 8.56(\mathrm{~m}, 1 \mathrm{H}), 8.55(\mathrm{~s}, 1 \mathrm{H}), 5.80(\mathrm{~d}$, $J=15.0 \mathrm{~Hz}, 1 \mathrm{H}), 5.12(\mathrm{~d}, J=14.9 \mathrm{~Hz}, 1 \mathrm{H}), 4.96(\mathrm{td}, J=$ 12.4, 4.1 Hz, 1H), 4.16 (d, $J=17.1 \mathrm{~Hz}, 1 \mathrm{H}), 3.69$ (d, $J=$ $17.1 \mathrm{~Hz}, 1 \mathrm{H}), 3.50(\mathrm{t}, J=4.1 \mathrm{~Hz}, 1 \mathrm{H}), 3.40(\mathrm{~d}, J=3.3 \mathrm{~Hz}$, $1 \mathrm{H}), 3.33$ (t, $J=4.3 \mathrm{~Hz}, 1 \mathrm{H}), 2.93$ (s, 3H), 2.79 (t, $J=7.0$ $\mathrm{Hz}, 1 \mathrm{H}), 2.42(\mathrm{~s}, 3 \mathrm{H}) ;{ }^{13} \mathrm{C}$ NMR (100 MHz, Pyridine- $\left.d_{5}\right) \delta$ : $172.57,151.03,166.79,158.38,131.14,128.54,121.02$, $120.96,119.42,104.05,56.07,51.75,50.88,46.33,37.09$, 30.16, 29.22, 22.04, 21.30; HRMS calcd for $\mathrm{C}_{19} \mathrm{H}_{24} \mathrm{~N}_{3} \mathrm{O}_{4} \mathrm{~S}_{2}$ $[\mathrm{M}+\mathrm{H}]^{+}$422.1204, found 422.1208.

(2-(2-(4-(3-差基苯基)噻唑-2-基)亚肼基)-7,7-二甲基 双环 [2.2.1] 庚-1-基) 甲磺酸 (Q17)：产率 80\%. m.p. $283.9 \sim 284.3{ }^{\circ} \mathrm{C} ;{ }^{1} \mathrm{H}$ NMR (400 MHz, Pyridine- $\left.d_{5}\right) \delta$ : $9.44(\mathrm{~s}, 1 \mathrm{H}), 8.39$ (d, $J=7.7 \mathrm{~Hz}, 1 \mathrm{H}), 8.31(\mathrm{~s}, 1 \mathrm{H}), 8.09$ (t, $J=7.9 \mathrm{~Hz}, 1 \mathrm{H}), 7.94$ (s, 1H), 5.04 (d, $J=15.0 \mathrm{~Hz}, 1 \mathrm{H})$, 4.35 (d, $J=15.0 \mathrm{~Hz}, 1 \mathrm{H}), 4.16$ (t, $J=12.2 \mathrm{~Hz}, 1 \mathrm{H}), 3.44$ $(\mathrm{d}, J=16.9 \mathrm{~Hz}, 1 \mathrm{H}), 2.96$ (d, $J=17.0 \mathrm{~Hz}, 1 \mathrm{H}), 2.73$ (t, $J=$ $11.5 \mathrm{~Hz}, 1 \mathrm{H}), 2.61$ (s, 2H), 2.15 (s, 3H), 1.94 (s, 1H), 1.63 $(\mathrm{s}, 3 \mathrm{H}) ;{ }^{13} \mathrm{C} \mathrm{NMR}\left(100 \mathrm{MHz}\right.$, Pyridine- $\left.d_{5}\right) \delta$ : 171.44, $164.35,159.78,152.27,138.03,130.59,117.89,116.00$, $114.62,103.93,54.76,50.68,49.73,45.23,35.70,29.06$, 28.14, 20.89, 20.15; HRMS calcd for $\mathrm{C}_{19} \mathrm{H}_{24} \mathrm{~N}_{3} \mathrm{O}_{4} \mathrm{~S}_{2}[\mathrm{M}+$ $\mathrm{H}]^{+}$422.1202, found 422.1208 .

(2-(2-(4-(4-差基苯基)噻唑-2-基)亚肼基)-7,7-二甲基 双环 [2.2.1] 庚-1-基) 甲磺酸 (Q18): 产率 88\%. m.p. $287.0 \sim 287.6{ }^{\circ} \mathrm{C} ;{ }^{1} \mathrm{H}$ NMR $\left(400 \mathrm{MHz}\right.$, Pyridine- $\left.d_{5}\right) \delta$ : $8.73(\mathrm{~s}, 1 \mathrm{H}), 8.09$ (d, $J=8.7 \mathrm{~Hz}, 2 \mathrm{H}), 7.60$ (s, 1H), 6.98 (s, $1 \mathrm{H}), 4.35$ (d, $J=15.0 \mathrm{~Hz}, 1 \mathrm{H}), 3.88$ (q, $J=7.0 \mathrm{~Hz}, 1 \mathrm{H})$, 3.65 (d, $J=15.0 \mathrm{~Hz}, 1 \mathrm{H}), 3.46$ (s, 1H), 2.75 (d, $J=17.0$ $\mathrm{Hz}, 1 \mathrm{H}), 2.28$ (d, $J=17.0 \mathrm{~Hz}, 1 \mathrm{H}), 1.91(\mathrm{~d}, J=4.0 \mathrm{~Hz}$, $2 \mathrm{H}), 1.45(\mathrm{~s}, 3 \mathrm{H}), 1.34 \sim 1.29(\mathrm{~m}, 1 \mathrm{H}), 0.97 \sim 0.90(\mathrm{~m}$, $3 \mathrm{H}) ;{ }^{13} \mathrm{C}$ NMR (100 MHz, Pyridine- $\left.d_{5}\right) \delta: 172.91,165.58$, 160.72 , 153.94, 129.92, 129.65, 118.36, 102.53, 56.26, 
$52.23,51.22,46.78,37.21,30.61,29.66,22.44,21.66$; HRMS calcd for $\mathrm{C}_{19} \mathrm{H}_{24} \mathrm{~N}_{3} \mathrm{O}_{4} \mathrm{~S}_{2}[\mathrm{M}+\mathrm{H}]^{+}$422.1204, found 422.1208.

(2-(2-(4-(4-氧基苯基)噻唑-2-基)亚肼基)-7,7-二甲基 双环 [2.2.1]庚-1-基) 甲磺酸 (Q19): 产率 93.6\%. m.p. $269.7 \sim 270.2{ }^{\circ} \mathrm{C} ;{ }^{1} \mathrm{H}$ NMR (400 MHz, DMSO- $\left.d_{6}\right) \delta$ : $8.11 \sim 8.00(\mathrm{~m}, 2 \mathrm{H}), 7.93 \sim 7.88(\mathrm{~m}, 1 \mathrm{H}), 7.85(\mathrm{~d}, J=8.5$ $\mathrm{Hz}, 1 \mathrm{H}), 7.57$ (s, 1H), 3.26 (d, $J=14.9 \mathrm{~Hz}, 1 \mathrm{H}), 2.72$ (d, $J=14.9 \mathrm{~Hz}, 1 \mathrm{H}), 2.49 \sim 2.44(\mathrm{~m}, 1 \mathrm{H}), 2.12 \sim 2.00(\mathrm{~m}, 1 \mathrm{H})$, 1.90 (s, 1H), 1.79 (s, 1H), 1.47 (s, 1H), 1.21 (d, $J=3.3 \mathrm{~Hz}$, 1H), $1.17(\mathrm{~s}, 1 \mathrm{H}), 1.05(\mathrm{~s}, 3 \mathrm{H}), 0.74(\mathrm{~s}, 3 \mathrm{H}) ;{ }^{13} \mathrm{C} \mathrm{NMR}$ $\left(100 \mathrm{MHz}, \mathrm{DMSO}-d_{6}\right) \delta: 169.69,146.30,146.00,137.34$, $132.67,132.67,126.19,126.19,118.83,109.94,107.16$, 53.68, 49.20, 48.87, 43.39, 34.66, 27.71, 26.65, 19.56, 19.25; HRMS calcd for $\mathrm{C}_{20} \mathrm{H}_{23} \mathrm{~N}_{4} \mathrm{O}_{3} \mathrm{~S}_{2} \quad[\mathrm{M}+\mathrm{H}]^{+}$ 431.1213, found 431.1211

(2-(2-(4-(4-联苯基)噻唑-2-基)亚肼基)-7,7-二甲基双 环 [2.2.1] 庚-1- 基) 甲磺酸 (Q20): 产率 89.6\%. m.p. $257.4 \sim 258.0{ }^{\circ} \mathrm{C} ;{ }^{1} \mathrm{H}$ NMR (400 MHz, Pyridine- $d_{5}$ ) $\delta$ : $9.73(\mathrm{~s}, 2 \mathrm{H}), 9.20$ (d, $J=8.4 \mathrm{~Hz}, 2 \mathrm{H}), 8.71(\mathrm{~s}, 1 \mathrm{H}), 8.60$ (s, $2 \mathrm{H}), 8.47$ (t, $J=7.6 \mathrm{~Hz}, 2 \mathrm{H}), 8.37$ (d, $J=7.4 \mathrm{~Hz}, 1 \mathrm{H}), 5.38$ (d, $J=15.0 \mathrm{~Hz}, 1 \mathrm{H}), 4.68(\mathrm{~d}, J=15.0 \mathrm{~Hz}, 1 \mathrm{H}), 4.50$ (t, $J=$ $10.1 \mathrm{~Hz}, 1 \mathrm{H}), 3.79$ (d, $J=16.9 \mathrm{~Hz}, 1 \mathrm{H}), 3.31(\mathrm{~d}, J=17.0$ $\mathrm{Hz}, 1 \mathrm{H}), 3.04$ (s, 1H), 2.92 (s, 2H), 2.48 (s, 3H), 2.32 (s, 1H), $1.96(\mathrm{~s}, 3 \mathrm{H}) ;{ }^{13} \mathrm{C}$ NMR $\left(100 \mathrm{MHz}\right.$, Pyridine- $\left.d_{5}\right) \delta$ : $171.63,164.45,151.71,141.43,140.57,135.63,129.76$, 129.76, 128.18, 127.94, 127.94, 127.64, 127.64, 127.36, 127.36, 104.20, 54.81, 50.70, 49.76, 45.26, 35.77, 29.09, 28.16, 20.93, 20.18; HRMS calcd for $\mathrm{C}_{25} \mathrm{H}_{28} \mathrm{~N}_{3} \mathrm{O}_{3} \mathrm{~S}_{2}[\mathrm{M}+$ $\mathrm{H}]^{+}$482.1573, found 482.1572 .

(2-(2-(4-(2-萗基)噻唑-2-基)亚肼基)-7,7-二甲基双环 [2.2.1]庚-1-基)甲磺酸(Q21): 产率 91.6\%. m.p. 279.6 $280.2{ }^{\circ} \mathrm{C} ;{ }^{1} \mathrm{H}$ NMR (400 MHz, DMSO- $\left.d_{6}\right) \delta: 8.48(\mathrm{~s}, 1 \mathrm{H})$, $8.11(\mathrm{q}, J=8.8 \mathrm{~Hz}, 1 \mathrm{H}), 7.97(\mathrm{~s}, 1 \mathrm{H}), 7.95 \sim 7.90(\mathrm{~m}, 2 \mathrm{H})$, $7.63 \sim 7.50(\mathrm{~m}, 2 \mathrm{H}), 7.47(\mathrm{~s}, 1 \mathrm{H}), 3.27(\mathrm{~d}, J=14.6 \mathrm{~Hz}$, $1 \mathrm{H}), 2.78(\mathrm{~d}, J=14.7 \mathrm{~Hz}, 1 \mathrm{H}), 2.58$ (s, 1H), 2.11 (d, $J=$ $18.1 \mathrm{~Hz}, 1 \mathrm{H}), 1.96(\mathrm{~s}, 1 \mathrm{H}), 1.84$ (s, 1H), $1.63(\mathrm{~s}, 1 \mathrm{H}), 1.25$ (s, 1H), $1.19 \sim 1.10(\mathrm{~m}, 1 \mathrm{H}), 1.03(\mathrm{~s}, 3 \mathrm{H}), 0.78(\mathrm{~s}, 3 \mathrm{H}) ;{ }^{13} \mathrm{C}$ NMR (100 MHz, Pyridine- $\left.d_{5}\right) \delta: 171.60,164.37,152.04$, $134.74,134.04,133.84,129.08,128.94,128.59,127.17$, $126.65,125.57,125.25,104.67,54.83,50.72,49.76,45.31$, $35.75,29.12,28.18,20.97,20.18$; HRMS calcd for $\mathrm{C}_{23} \mathrm{H}_{26} \mathrm{~N}_{3} \mathrm{O}_{3} \mathrm{~S}_{2}[\mathrm{M}+\mathrm{H}]^{+}$456.1417, found 456.1415

(2-(2-(4-苯基)噻唑-2-基)亚肼基)-7,7-二甲基双环 [2.2.1]庚-1-基)甲磺酸(Q22): 产率 96.8\%. m.p. 292.5
$293.1{ }^{\circ} \mathrm{C} ;{ }^{1} \mathrm{H}$ NMR (400 MHz, DMSO- $\left.d_{6}\right) \delta: 7.83(\mathrm{~d}, J=$ $7.2 \mathrm{~Hz}, 2 \mathrm{H}), 7.42(\mathrm{t}, J=7.3 \mathrm{~Hz}, 2 \mathrm{H}), 7.36(\mathrm{t}, J=7.2 \mathrm{~Hz}$, 1H), 7.29 (s, 1H), 3.23 (d, $J=14.7 \mathrm{~Hz}, 1 \mathrm{H}), 2.74$ (d, $J=$ $14.7 \mathrm{~Hz}, 1 \mathrm{H}), 2.54$ (s, 1H), 2.44 (s, 1H), 2.09 (d, $J=18.1$ $\mathrm{Hz}, 1 \mathrm{H}), 1.93$ (t, $J=4.0 \mathrm{~Hz}, 1 \mathrm{H}), 1.83(\mathrm{~s}, 1 \mathrm{H}), 1.58(\mathrm{~s}, 1 \mathrm{H})$, 1.23 (s, 1H), 1.03 (s, 3H), $0.76(\mathrm{~s}, 3 \mathrm{H}) ;{ }^{13} \mathrm{C}$ NMR (100 $\mathrm{MHz}$, Pyridine- $d_{5}$ ) $\delta: 171.46,164.25,151.89,136.38$, 129.26, 128.06, 126.67, 103.78, 54.64, 50.54, 49.58, 45.10, $35.58,28.92,27.99,20.75,20.00$; HRMS calcd for $\mathrm{C}_{19} \mathrm{H}_{24}$ $\mathrm{N}_{3} \mathrm{O}_{3} \mathrm{~S}_{2}[\mathrm{M}+\mathrm{H}]^{+}$406.1247, found 406.1259.

\section{4 抗氧化活性测定}

\subsubsection{DPPH 自由基清除实验}

根据文献报道 ${ }^{[13]}$, 在 96 孔酶标板中加入 $200 \mu \mathrm{L}$ $\mathrm{DPPH}$ 的乙醇溶液 $(100 \mu \mathrm{mol} / \mathrm{L})$, 然后将 $50 \mu \mathrm{L}$ 等分式样 的不同浓度的药液以及对照组(以去离子水代替药液)分 别加入每个孔中. 在 $37{ }^{\circ} \mathrm{C}$ 温育 $30 \mathrm{~min}$ 后, 使用酶标仪 在 $490 \mathrm{~nm}$ 处测定每种溶液的光密度.

$$
\text { 抑制率 }(\%)=\left[1-\frac{\mathrm{OD}_{\text {实验 }}-\mathrm{OD}_{\text {空白白 }}}{\mathrm{OD}_{\text {对照 }}-\mathrm{OD}_{\text {空白 }}}\right] \times 100 \%
$$

其中: $\mathrm{OD}$ 实验为测试药物的平均光密度, $\mathrm{OD}$ 对照为对照组 的平均光密度, $\mathrm{OD}$ 空白为空白组的平均光密度.

\subsubsection{ABTS 阳离子自由基清除实验}

根据文献报道的方法 ${ }^{[12]}$ 进行 $\mathrm{ABTS}$ 自由基消除实 验. 在蒸馏水中以体积比为 $2: 1$ 混合 $7 \mathrm{mmol}$ ABTS 和 $2.45 \mathrm{mmol}$ 过硫酸钾, 在黑暗中氧化 $12 \sim 16 \mathrm{~h}$ 制得 ABTS 实验溶液. 氧化结束后, 使用蒸馏水稀释使得 ABTS 溶液在 $734 \mathrm{~nm}$ 下吸光度为 $(0.700 \pm 0.020)$. 将 50 $\mu \mathrm{L}$ 等分试样的不同浓度的药液和对照组(以去离子水代 替药液)分别加入含有 $200 \mu \mathrm{L} \mathrm{ABTS}$ 溶液的 96 孔板的每 个孔中, 在 $37{ }^{\circ} \mathrm{C}$ 温浴 $10 \mathrm{~min}$ 后, 使用酶标仪在 $734 \mathrm{~nm}$ 处测定每种溶液的光密度.

\subsection{3 过氧阴离子自由基清除实验}

根据文献[14], 以抗氧化剂对 DCFH 氧化的抑制程 度作为清除过氧自由基的抗氧化活性. 因为 DCFH-DA 较稳定, 不易被氧化, 而 DCFH 在 ABAP 的环境中易被 氧化. 所以在反应之前, 以如下方法配置 DCFH 溶液待 用: 将 $80 \mu \mathrm{L}$ 的 $2.48 \mathrm{mmol}$ DCFH-DA 在小瓶中用 $900 \mu \mathrm{L}$ 的 $1.0 \mathrm{mmol} \mathrm{KOH}$ 水解 3 5 min 以除去二乙酸酯(DA) 部分, 然后稀释至 $6 \mathrm{~mL}$. 在 96 孔酶标板中加入 $100 \mu \mathrm{L}$ 配置好的 DCFH 溶液, 将 $100 \mu \mathrm{L}$ 等分式样的不同浓度 的药液以及对照组(以去离子水作为阴性对照, 水溶性 维生素 $\mathrm{E}$ 作为阳性对照)分别加入每个孔中, 在 1200 $\mathrm{r} / \mathrm{min}$ 振荡 $20 \mathrm{~s}$ 将每个孔中的溶液混匀, 然后在每个孔 中加入 $50 \mu \mathrm{L} \mathrm{AAPH}$ 溶液 $(40 \mathrm{mmol})$ 引发反应. 每种抑制 
剂的每个浓度均做三个平行对照. 反应在 $37{ }^{\circ} \mathrm{C}$ 下进行, 使用酶标仪在 $485 \mathrm{~nm}$ 激发光和 $538 \mathrm{~nm}$ 发射光下检测荧 光强度变化 $40 \mathrm{~min}$. 以抑制剂的平均荧光与反应时间动 力学曲线下的面积积分来计算抑制剂的过氧自由基清 除能力.

\subsection{4 羟基自由基还原能力测定}

羟基自由基是重要的活性氧物质. 羟基自由基具有 非常强的电子获得能力, 即氧化能力. 使用着自由基试 剂盒来测定樟脑磺酸噻唑腙类化合物在浓度为 $4 \mathrm{mmol}$ 的羟自由基清除能力.

\subsection{5 对酪氨酸酶抑制率测定}

根据参考文献描述的方法 ${ }^{[16]}$ ，使用左旋多巴作为 底物评价了化合物 $\mathbf{Q} \sim \mathbf{Q 2 2}$ 对酪氨酸酶的抑制效果. 将 $100 \mu \mathrm{L}$ 的 $100 \mathrm{mmol}$ 磷酸缓冲液 ( $\mathrm{pH}$ 为 6.8 ) 和 $20 \mu \mathrm{L}$ 的 $250 \mathrm{U} / \mathrm{mL}$ 酪氨酸酶加入到 96 孔板的每个孔中, 然后 以 $40 \mu \mathrm{L}$ 等分试样的不同浓度的药液和对照组(以去离 子水代替药液)分别加入每个孔中, 在室温下温浴 15 $\mathrm{min}$, 温浴后向每个孔中加入 $20 \mu \mathrm{L}$ 的 $3 \mathrm{mmol}$ 左旋多巴 并开始反应. 使用酶标仪在 $475 \mathrm{~nm}$ 下测量吸光度.

辅助材料(Supporting Information) 中间体化合物 $\mathbf{2}$ 的 核磁共振氢谱、碳谱以及 HRMS 谱图; 最终产物 Q1 Q22 的核磁共振氢谱、碳谱图. 这些材料可以免费从本 刊网站(http://sioc-journal.cn/)上下载.

\section{References}

[1] Limtrakul, P.; Yodkeeree, S.; Thippraphan, P. BMC Complementary Altern. Med. 2016, 16, 497.

[2] Gragnani, A.; Cornick, S.; Chominski, V. Adv. Aging Res. 2014, 3, 265.

[3] Pedrosa, T.; Barros, A.; Nogueira, J. Arch. Dermatol. Res. 2016, $308,643$.

[4] Delalle-Lozica, N. Acta Clin. Croat. 2010, 49, 529.

[5] Jae, Y. L.; Young-Rak, C.; Ju, H. P. Toxicol. Rep. 2019, 6, 10

[6] Limtrakul, P.; Yodkeeree, S.; Thippraphan, P. BMC Complementary Altern. Med. 2016, 16, 497.

[7] Alfredo, G.; Sarita, M. C.; Verônica, C. Adv. Aging Res. 2014, 3,
265.

[8] Laguerre, M.; Lecomte, J.; Villeneuve, P. Prog. Lipid Res. 2007, 46, 244.

[9] Amorati, R.; Foti, M.; Valgimigli, L. J. Agric. Food Chem. 2013, $61,10835$.

[10] Liu, Y.; Huang, G.; Hu, J. Int. J. Biol. Macromol. 2018, 111, 1304.

[11] Didem, Ş.; Suat, S.; Burak, B. Bioorg. Chem. 2018, 81, 168.

[12] Li, X.; Huang, J.; Wang, Z. Sep. Purif. Technol. 2014, 124, 201.

[13] Adelakun, O.; Kudanga, T.; Parker, A. J. Mol. Catal. B: Enzym. 2012, 74, 29 .

[14] Adom, K.; Liu, R. J. Agric. Food Chem. 2005, 53, 6572.

[15] Erzsébet, I.; Amir, M.; Vered, M. Free Radical Biol. Med. 2019, 131,1 .

[16] Jae, Y.; Young, R.; Ju, H. Toxicol. Rep. 2019, 6, 10.

[17] Olanow, C. Trends Neurosci. 1993, 16, 439.

[18] Bennett, S.; Grant, M.; Aldreda, S. J. Alzheimer's Dis. 2009, 17, 245.

[19] Rietveld, I.; Barrio, M.; Veglio, N. Thermochim. Acta 2010, 511, 43.

[20] Andersson, O.; Ross, R.; Jezowski, A. Mol. Phys. 1990, 70, 1065.

[21] Kisiel, Z.; Desyatnyk, O.; Białkowska-Jaworska, E. Phys. Chem. Chem. Phys. 2003, 5, 820.

[22] Hargrave, K.; Hess, F.; Oliver, J. J. Med. Chem. 1983, 26, 1158.

[23] Patt, W.; Hamilton, H.; Taylor, M. J. Med. Chem. 1992, 35, 2562.

[24] Kamble, R.; Meshram, R.; Hese, S. Comput. Biol. Chem. 2016, 61, 86.

[25] Jaen, J.; Wise, L.; Caprathe, B. J. Med. Chem. 1990, 33, 311.

[26] Mohammad, H.; Reddy, P.; Monteleone, D. Eur. J. Med. Chem. 2015, 94, 306

[27] Ergenç, N.; Çapan, G.; Günay, N. Arch. Pharm. (Weinheim, Ger.) 1999, 332, 343.

[28] Bell, F.; Cantrell, A.; Hoegberg, M. J. Med. Chem. 1995, 38, 4929.

[29] Badorc, A.; Bordes, M.; Cointet, P. Med. Chem. 1997, 40, 3393.

[30] Khan, K.; Qurban, S.; Salar, U. Bioorg. Chem. 2016, 68, 245.

[31] Wang, G.; Peng, Z.; Gong, Z. Bioorg. Chem. 2018, 78, 195.

[32] Paudel, Y. N.; Ali, M. R.; Shah, S. Biomed. Pharmacother. 2017, $89,651$.

[33] Rui, J.; Zhang, Q.; Wang, X. Chin. J. Org. Chem. 2017, 37, 218 (in Chinese).

(茌坚, 张齐, 王欣, 有机化学, 2017, 37, 218.)

[34] Sun, N.; Wang, X.; Ding, Z. Chin. J. Org. Chem. 2016, 36, 2489. (in Chinese). (孙楠, 王欣, 丁志涁, 有机化学, 2016, 36, 2489.)

[35] Wang, Y.; Gu, W.; Shan, Y. Bioorg. Med. Chem. Lett. 2017, 27, 2360.

[36] Yang, J.; Xu, X.; Rui, J. Spectrochim. Acta, Part A 2017, 183, 60.

[37] Yang, J.; Xu, H.; Xu, X. Dyes Pigm. 2016, $128,75$.

[38] Vinícius, G. M.; Marina, F. R.; Thales, K. J. Mol. Graphics Modell. 2019, 86, 106. 